\title{
National survey on the roles and training of primary care respiratory nurses in the UK in 2006: are we making progress?
}

\section{*Jane Upton ${ }^{\text {, }}$ Hazel Madoc-Sutton ${ }^{\text {b }}$ Aziz Sheikhc, Timothy L Frank ${ }^{\text {d }}$, Samantha Walkere, Monica Fletcherf}

\author{
a Research Project Manager, Education for Health, 10 Church Street, Warwick, CV34 4AB, UK \\ ${ }^{b}$ Research Assistant, Education for Health, Warwick, UK \\ Professor of Primary Care Research and Development, Allergy and Respiratory Research Group, Division of Community Health Sciences: \\ GP Section, University of Edinburgh, Scotland, UK \\ 'Director, General Practice Research Unit, North West Lung Research Centre, Wythenshawe Hospital, Manchester, UK \\ e Director of Education and Research, Education for Health, Warwick, UK \\ ${ }^{\dagger}$ Chief Executive, Education for Health, Warwick, UK
}

Received 6th August 2007; accepted 3rd September 2007

\begin{abstract}
Objectives: To describe nurse-led UK general practice asthma and chronic obstructive pulmonary disease (COPD) care, and the training undertaken to support it.

Methods: Questionnaires were sent to 500 randomly-selected UK asthma and COPD practice nurses.

Results: 382 nurses (76\%) completed the practice characteristics section, 389 (78\%) described their asthma roles and training, and 368 (74\%) described their COPD roles and training. 96 practices (25\%; 95\% Cl 21-29\%) ran designated asthma clinics, 87 (23\%; $95 \% \mathrm{Cl} 19$ $27 \%$ ) ran designated COPD clinics, and $170(45 \% ; 95 \% \mathrm{Cl} 40-49 \%)$ did not run designated respiratory clinics. Of the 255 nurses with an advanced asthma role, $51(20 \% ; 95 \% \mathrm{Cl} 15-25 \%)$ did not have accredited asthma training. Of the 215 nurses with an advanced COPD role, $111(52 \% ; 95 \% \mathrm{Cl} 45-58 \%)$ did not have accredited COPD training.

Conclusion: Patients are increasingly being seen outside of designated asthma or COPD clinics, often by nurses with an advanced role. it is important that nurses have the training to fulfil this role.

(c) 2007 General Practice Airways Group. All rights reserved.

J Upton, et al. Prim Care Resp J 2007; 16(5): 284-290.

doi:10.3132/pcrj.2007.00068
\end{abstract}

Keyw ords asthma, COPD, general practice, practice nurses, education

\section{Introduction}

In the UK, most patients with airways diseases are now managed in primary care by practice nurses working in partnership with their general practitioner (GP) colleagues. This constitutes a major change in the delivery of care compared to 20 years ago, and it was driven initially by the introduction of a new GP contract in 1990. ' This encouraged health education, and systematic care and regular review of patients with asthma, diabetes, and coronary heart disease risk factors such as smoking and obesity, by introducing a payment system for running disease clinics. At that time, chronic obstructive pulmonary disease (COPD) was not widely managed in primary care and was therefore not included in the 1990 GP contract.

Much of this chronic disease management could be performed by appropriately trained nurses following agreed guidelines. ${ }^{2}$ As a result of these changes the number of practice nurses rapidly increased ${ }^{3}$ and their roles developed to meet these new contractual requirements. The extent of an individual nurse's responsibility for chronic disease

*Corresponding author: Tel: +44 (0)1926 838976 Fax: +44 (0)1926 493224 E-mail: j.upton@educationforhealth.org.uk 
management in clinics varied from practice to practice, ranging from a supporting role with the GP, to taking full responsibility without support from a GP. GPs began to recognise that practice nurses could play a key role in delivering asthma services, and most were happy to allow the nurse to assume an extended role provided they had appropriate training. ${ }^{4}$ Nurses also demanded this training, and practice nurses across the UK, in conjunction with the Royal College of Nursing Practice Nurse Association, lobbied for specialist qualifications. ${ }^{5}$

The contractual arrangements for UK GPs were revised again in $1993 .{ }^{6}$ In these revisions the need to run designated clinics was removed, and GPs received a fixed annual payment for running organised programmes of care for patients with chronic diseases. By the late 1990's COPD had become increasingly managed and diagnosed within primary care. Many nurses who had previously been responsible for running asthma care began to expand their roles into COPD management. National consensus guidelines ${ }^{7}$ and accredited training courses became available which supported this clinical development.

The introduction of the General Medical Services (GMS) contract of $2003^{8}$ changed the landscape of primary care in the UK yet again. Practices were financially rewarded for achieving quality indicators (i.e. clinical achievement data) for managing asthma and COPD.

A National Service Framework for COPD ${ }^{9}$ is due to be published imminently. This is likely to recognise the role that primary care nurses play in the management of COPD. However, although much of this work continues to be delegated to nurses, their levels of training and competencies are not monitored or audited outside of the employing practice. This presents clear clinical governance concerns.

Nurses' roles are therefore changing to reflect the developments in patient care occurring within the NHS, and it is important to ensure that nurses have the required training and competencies required in these roles. This survey describes the organisation of asthma and COPD care in UK general practice in 2006. It also investigates the roles that practice nurses have adopted in the management of airways disease, and the training they have undertaken to prepare themselves for these roles.

\section{Methods}

The survey was conducted between February and June 2006. The contact details of all UK general practices $(\mathrm{N}=10,419)$ were obtained from the Binley's database (details available at: http://www.binleys.com). The target sample size of 1537 was calculated using a level of precision of $\pm 5 \%$. Three thousand general practices were selected through simple random sampling and were sent a pre-piloted questionnaire (details below) together with a pre-paid envelope. It was requested that the nurse who routinely looked after patients with asthma complete the asthma section, and that the nurse who routinely looked after patients with COPD complete the COPD section. The questionnaires were followed by up to two reminder letters to non-responders, sent two weeks apart.

Despite these three mailings, the overall response rate was poor $(21 \%)$. In order to obtain a higher response rate, we therefore conducted a nested survey. Using a level of precision of $\pm 10 \%$, the target sample size was calculated as being 371 respondents. Five hundred respondents were randomly selected from the original 3000 general practices, and non-responders were contacted and reminded by telephone to return their questionnaire. This paper reports the results of this nested survey.

Ethical approval was not required as this survey was categorised as a service evaluation. However, all relevant Primary Care Trusts in England, Health Boards and Local Health and Social Care Groups in Northern Ireland, Local Health Boards in Wales and Community Health Partnerships in Scotland were advised that the survey was being conducted.

\section{Details of the questionnaire}

The questionnaire included three sections: a practice characteristic section (which included practice list size and types of designated respiratory clinics run by the practice); a section recording the role and training of the lead asthma nurse(s); and a section recording the role and training of the lead COPD nurse(s). Respondents were asked to report if their practice ran one or more of the following types of designated respiratory clinic: asthma, COPD, and/or mixed respiratory. Respondents were also given the option "none, patients seen in generic appointments". The questionnaire included a list of key tasks performed during consultations, and respondents were asked to identify which person normally performed each type of task. Nurses were also required to state all relevant training they had obtained.

\section{Analysis}

Questionnaires were excluded if the asthma and COPD sections had been completed by the GP; this was because the survey aimed to report the care provided and training undertaken by practice nurses. Descriptive statistics were generated using Minitab system 13.31.

Nursing roles were divided into three levels:

1. Basic: the nurse does not autonomously give follow-up care or autonomously confirm diagnosis.

2. Intermediate:

a. The nurse autonomously gives follow-up care (but does not autonomously confirm diagnosis) or

b. The nurse autonomously confirms diagnosis (but does not autonomously give follow-up care). 
3. Advanced: The nurse autonomously gives follow-up care AND autonomously confirms diagnosis.

Types of training were categorised as being either accredited (diploma and degree level modules recognised by a university) or non-accredited (short courses, pharmaceutical company sponsored meetings and in-house training). Data were also analysed to ascertain the proportion of nurses without accredited training: i) by role level and ii) by practice size.

\section{Results}

Response rates obtained for the smaller nested sub-survey were: $76 \%$ (382/500) for the practice characteristic section; $78 \%$ (389/500) for the asthma section; and 74\% (368/500) for the COPD section of the questionnaire.

General practices from throughout the UK were included in the survey. Of the 382 practices that completed the practice characteristic section of the questionnaire, 307 (80\%) were based in England, $46(12 \%)$ were based in Scotland, 15 (4\%) were based in Wales, and 14 (4\%) were based in Northern Ireland.

\section{Figure 1. Distribution of practice list size.}

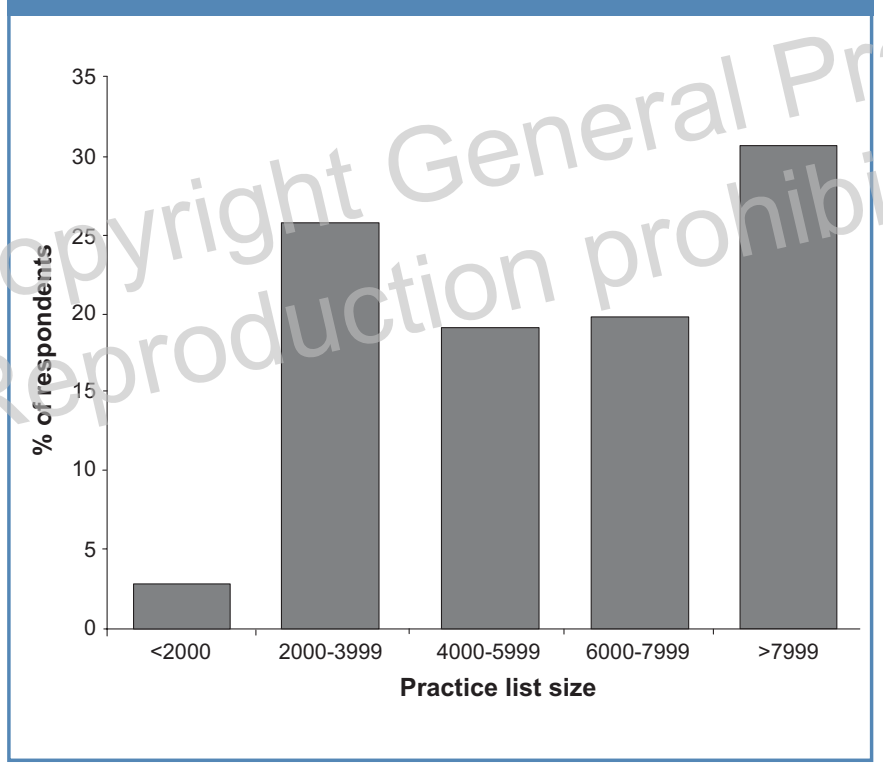

\section{Practice list size and types of clinics}

Practices were grouped by practice list size. The proportion of practices in each group is shown in Figure 1. Only $3 \%$ of the respondents (13/382) were based in practices with less than 2000 patients.

Of the 382 practice nurses who completed the practice characteristic section of the questionnaire, 96 practices (25\%; $95 \% \mathrm{Cl} 21-29 \%$ ) ran a designated asthma clinic, 87 practices (23\%; $95 \% \mathrm{Cl} 19-27 \%)$ ran a designated COPD clinic, and 72 practices $(19 \% ; 95 \% \mathrm{Cl} 15-23 \%)$ ran a mixed respiratory clinic. One hundred and seventy nurses $(45 \% ; 95 \% \mathrm{Cl} 40$ $49 \%)$ reported that their general practice did not see patients in designated respiratory clinics.

A higher proportion of the larger general practices - as compared to the smaller practices - ran designated respiratory clinics: $63 \%(\mathrm{~N}=169)$ of practices with list sizes of 4000 or more patients ran clinics compared with $37 \%(N=42)$ of smaller practices.

\section{Role and training of practice nurses}

The proportions of practice nurses at each role level are shown in Table 1. Over half of the nurses providing asthma or COPD care were working in an advanced role and approximately one-third held an intermediate role.

Figure 2 shows that, of the 255 respondents who held an advanced role in the care of patients with asthma, 51 (20\%; $95 \% \mathrm{Cl} 15-25 \%)$ did not have accredited asthma training, of whom $21(41 \%)$ reported that a GP was not always immediately accessible for advice if required when they were seeing patients.

Two hundred and fifteen respondents reported that they held an advanced role in the care of patients with COPD. Of these, 111 (52\%; 95\% Cl 45-58\%) had not obtained accredited COPD training (see Figure 2) and 198 (92\%; $95 \% \mathrm{Cl} 88-96 \%)$ had not obtained accredited spirometry training. Fifty of the 111 nurses (45\%) holding an advanced COPD role, but no accredited COPD training, reported that a GP was not always immediately accessible when they were seeing patients.

Most of the nurses holding an advanced role, and a high proportion of those holding an intermediate role,

Table 1. Role of nurses.

\begin{tabular}{|c|c|c|c|c|c|c|c|}
\hline & \multicolumn{6}{|c|}{ Role } & \multirow{3}{*}{ Total } \\
\hline & \multirow{2}{*}{\multicolumn{2}{|c|}{$\begin{array}{l}\text { Basic } \\
\%(95 \% \mathrm{Cl})\end{array}$}} & \multicolumn{2}{|c|}{ Intermediate } & \multicolumn{2}{|c|}{ Advanced } & \\
\hline & & & & $\%(95 \% \mathrm{Cl})$ & & $\%(95 \% \mathrm{Cl})$ & \\
\hline Asthma & 12 & $3(1-5)$ & 122 & $31(27-36)$ & 255 & $66(61-70)$ & $389(100)$ \\
\hline COPD $^{1}$ & 27 & $7(5-10)$ & 126 & 34 (29-39) & 215 & $58(53-63)$ & $368(100)$ \\
\hline
\end{tabular}




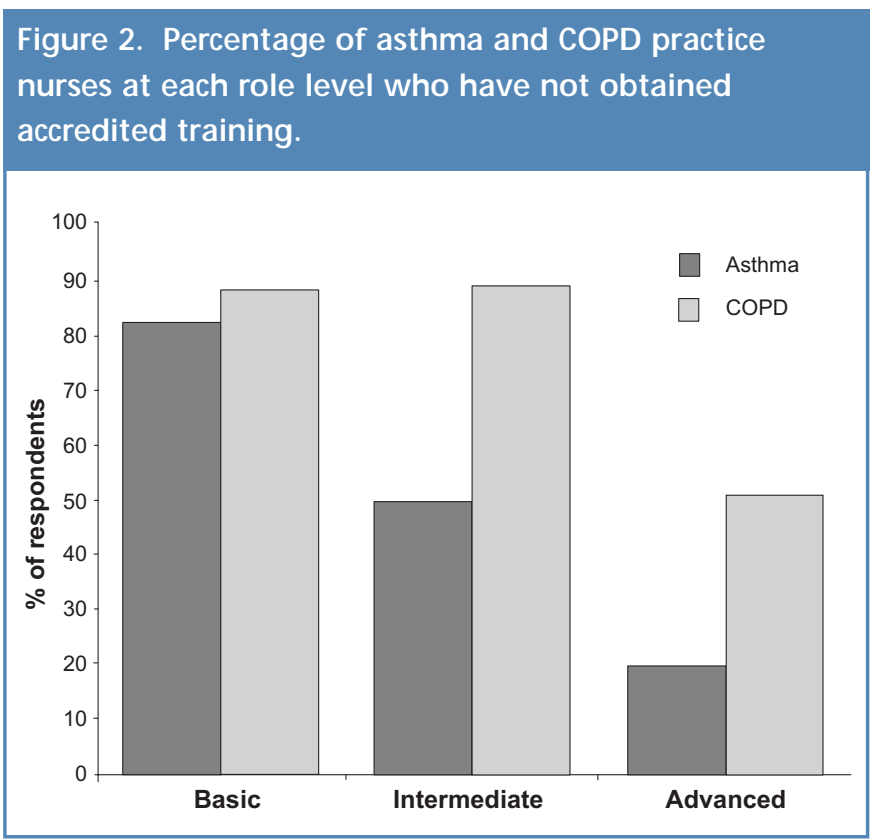

Table 2. Proportion of nurses holding an intermediate or advanced role who also recommend choices of therapy.

\begin{tabular}{l|c|c} 
& \multicolumn{2}{|c}{ Role } \\
\hline & Intermediate & Advanced \\
& $\mathrm{N}(\%)$ & $\mathrm{N}(\%)$ \\
\hline Asthma & $74 / 122(61)$ & $249 / 255(98)$ \\
\hline COPD & $62 / 126(49)$ & $205 / 215(95)$ \\
\hline
\end{tabular}

${ }^{1}$ Chronic obstructive pulmonary disease

(1)

Figure 3. The percentage of nurses without accredited training by practice list size.

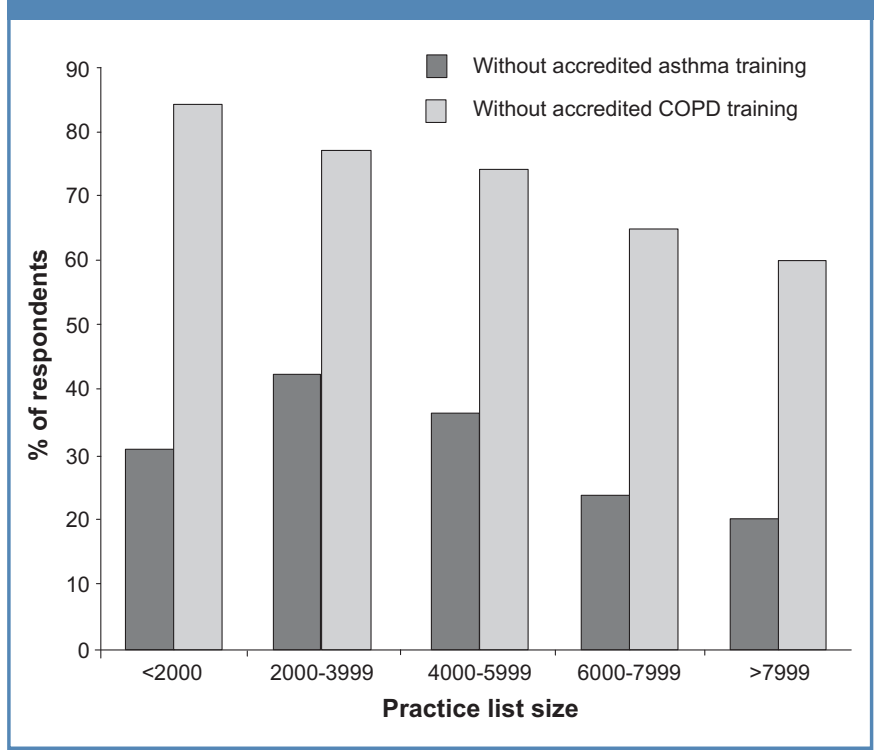

recommended choice of therapy during consultations (see Table 2). Of those nurses recommending choice of asthma therapies, 35\% (26/74) in an intermediate role and 19\% (47/249) in an advanced role did not have accredited training. Of nurses recommending choice of COPD therapies, $85 \%$ $(53 / 62)$ in an intermediate role and 50\% (102/205) in an advanced role did not have accredited training.

The proportion of nurses without accredited training was compared between practices with different list sizes (see Figure 3). For nurses caring for patients with asthma the training level did not vary widely by practice size - although the largest practices had the lowest proportion of nurses without accredited training (20\%). However, for nurses caring for COPD patients, the proportion of nurses without accredited COPD training was highest in the smallest practices (85\%), and decreased as practice list size increased.

\section{Discussion}

This survey aimed to investigate the organisation of asthma and COPD care in UK general practice, to look at the roles that practice nurses currently undertake in the management of airways disease, and to ascertain the level of training they have undertaken to prepare themselves for these roles.

\section{Practice list size and types of clinics}

Only $3 \%$ of respondents reported working in practices of less than 2000 patients. This proportion is smaller than a previous study conducted in 1993, in which it was reported that $9 \%$ of practices had a list size of less than 2000 patients. ${ }^{10}$ This decrease mirrors the trend in the national data available. ${ }^{11}$ It has been suggested that the increase in practice sizes may partly be due to doctors being attracted to working in larger practices, which allows scope for specialisation and potentially enables a wider range of services to be offered to patients. ${ }^{12}$

In this survey approximately two-thirds of practices with list sizes $\geq 4000$ and only one-third of practices with list sizes $<4000$ reported the running of designated respiratory clinics. The new GMS contract provides encouragement for organisational improvements which will support better chronic disease management. This may have encouraged some practices to run fewer designated respiratory clinics. More generic care may have been more easily embraced by smaller practices (in which the only practice nurse has historically provided all the chronic disease reviews) as compared to larger practices where the responsibilities are shared amongst the nursing staff.

This survey found that the number of designated asthma and designated COPD clinics has decreased since 1993, when these data were reported by the National Asthma Task Force. ${ }^{10}$ In 1993, $23 \%$ of practices saw patients with asthma 
in generic appointments; this current survey indicates that this percentage has risen, suggesting that asthma and COPD patients are increasingly being seen outside designated respiratory clinics. It is likely that the shift in organisation of care, away from designated respiratory clinics, has been mainly due to the changes in the GP and General Medical Services contract whereby GPs are not paid for running these designated clinics. This demonstrates that changes in remuneration and policy can affect the way in which care is delivered.

The best method of organising primary care respiratory services in order to optimise patient outcomes is unclear. ${ }^{13,14}$ Designated respiratory clinics may enable less experienced nurses to develop their clinical skills more rapidly since they are concentrating on one disease state. Conversely, generic appointments provide patients with more flexible access to practice nurses, and do not require patients with a number of long-term conditions to attend several different types of clinics.

Nurses are increasingly seeing patients outside designated respiratory clinics. The decrease in the number of these clinics means that they are increasingly seeing consecutive patients with different disease states. It must be ensured that nurses obtain sufficient training in order to provide a high standard of care. It is therefore important that this training reflects the needs of the workforce. This may require more holistic training to be provided (e.g. training nurses in shared-decision making and patient-centred care).

\section{Roles and training of practice nurses}

The drive to improve the management of long-term conditions in primary care has led to increased delegation of the care and management of these patients to practice nurses. This survey has found that two-thirds of asthma nurses undertake an advanced role in the care of patients $17 \%$ higher than reported in 1993. ${ }^{10}$

Most of these asthma and COPD nurses - with an advanced or intermediate role - recommended choices of therapy. This is likely to make services more accessible for patients and it widens patient choice. ${ }^{15}$ It has also been reported that nurses with appropriate training working in an advanced role provide a high level of patient care. ${ }^{16}$

However, this survey suggests that one-fifth of asthma nurses holding an advanced role do not have accredited training. This situation has not improved since 1993, when it was reported that $22 \%$ of nurses who were solely in charge of running an asthma clinic did not have accredited training. ${ }^{10}$

The level of COPD training was not reported in 1993. In 2006 approximately a half of COPD nurses holding an advance role did not have accredited training. This low level may be because this training has only been available for about a decade.
It would therefore appear from these survey findings that a large percentage of asthma and COPD nurses have not been trained appropriately. As $41 \%$ of asthma nurses and $45 \%$ of COPD nurses reported that a GP was not always immediately accessible when they were seeing patients, it would also appear that they are often not provided with sufficient supervision in order to undertake many of their duties.

The proportion of nurses without accredited asthma training was only slightly higher in the smallest practices participating in the survey (list size $<2000$ ) as compared to that in the largest practices (list size $>7999$ ). However, the proportion of nurses without accredited COPD training increased as practice list size decreased - suggesting that nurses based in small practices may not have the same level of COPD knowledge as nurses based in larger practice. There are two possible explanations for the difference (in COPD training levels) between smaller and larger practices. Firstly, nurses with specialist COPD training may be attracted to work at larger practices because the practices provide greater scope for clinical specialism. Secondly, small practices have difficultly releasing staff and therefore may not easily be able to provide access to this training. Since practice nurses working in smaller practices may see patients without the same level of support from colleagues as is available to nurses based in larger practices, it is important that these nurses have sufficient knowledge to care autonomously for patients with COPD.

This survey suggests that a large percentage of patients are currently being managed by nurses who are inadequately trained, and often not adequately supervised by their GP colleagues. At a time when the UK National Health Service (NHS) is focusing on the management of risk, this is potentially a serious failing within primary care, and needs to be addressed through clinical governance mechanisms.

\section{Strengths and limitations of this study}

The main difficulty encountered during the study was in obtaining a good response rate. The response rate to the initial 3000 questionnaires posted to practices was poor. We therefore decided to conduct a more limited survey of 500 practices, which could then be more intensively followed-up so as to achieve a good response rate. The final response rates achieved in this sub-sample were very high. The geographical distribution of responders reflects the location of practices across the UK. ${ }^{17}$ This, together with the survey's high response rate, yielded findings that are likely to be representative of UK general practice.

Only $3 \%$ of nurses who participated in the survey were employed by practices with list sizes of less than 2000. It is therefore possible that smaller practices are underrepresented in this survey. However, it is not possible to 
confirm this, as data from non-responders were not collected and were not provided by Binleys. To our knowledge national statistics do not specifically report the number of UK practices with list sizes of less than 2000 patients. It has previously been reported that small practices are less likely than larger practices to employ a practice nurse. ${ }^{18}$ As the present survey required a practice nurse to complete the questionnaire, it is possible that fewer responses were obtained from practices with less than 2000 patients than from larger practices. The findings may not therefore be representative of these small practices.

Respondents were asked if their surgery ran designated respiratory clinics or if patients were seen in 'generic appointments'. We intended the term 'generic appointments' to refer to appointments that took place in routine surgery (that is, outside designated respiratory clinics). A limitation of this term is that it might be interpreted by respondents in a number of different ways (e.g. patients seen in general consultations or in a generic long-term conditions clinic). However, as the emphasis of the survey was on the number of surgeries that ran designated respiratory clinics, the differing interpretations of this term do not affect the findings presented here i.e. the percentage of practices that ran these designated clinics.

\section{Clinical implications}

In recent years in the UK there have been several drives to improve the care and management of COPD..$^{19}$ In 1999 it was estimated that inpatient stays for COPD cost the UK NHS f243.4 million annually. ${ }^{20}$ Pressure on the NHS to reduce waiting times by preventing unnecessary admissions and shortening the number of bed days has made managers and providers look for ways of improving standards of care. The GMS contract of 2003 recognised that COPD was a significant long-term illness which was managed in primary care, and aimed to raise the standards of care provided by rewarding GPs for providing quality care. The GMS contract Quality and Outcomes Framework (QOF) does not currently stipulate the level of training a nurse should have before tasks are delegated to them. ${ }^{8}$ In order to improve the quality of services provided this should become a necessity in any future revisions of the contract. A COPD National Service Framework is imminent; ${ }^{9}$ this will further raise the profile of this condition.

A major shift has happened in the care of patients with COPD over the last decade, and COPD is now accepted as a disease that can be managed within primary care. It is important that this change is supported by adequate levels of training for nurses, many of whom are working without medical support. Accredited training must be provided for all nurses taking on these advanced roles within respiratory care in order to ensure high quality and safe patient care.

\section{Conclusions}

The large numbers of practice nurses working at an advanced role, most of whom are involved in therapy choices, indirectly indicates that progress has been made to improve respiratory care in primary care by utilising the practice nurse workforce. However, many nurses with an advanced role did not have accredited training and are under-supervised, particularly those working with COPD patients. This presents clinical governance issues, both for individual practices and for Primary Care Trusts. Patient care will not be optimal unless there is a suitably skilled workforce to undertake these increasingly responsible and demanding roles.

\section{Acknow ledgements}

We would like to acknowledge the help of Kim Esslemont whose assistance in collecting the data was invaluable. We would also like to thank Trisha Weller for commenting on this article.

\section{Conflict of interest declaration}

Jane Upton, Monica Fletcher, Samantha Walker and Hazel Madoc-Sutton are employed by a training organisation, Education for Health. Professor Aziz Sheikh is a medical research advisor for Education for Health; he is also an Assistant Editor of the PCRJ, but was not involved in the editorial review of, nor the decision to publish, this article.

Timothy Frank has received fees from GSK, Boehringer Ingelheim, Schering Plough and AstraZeneca for speaking, funds for research from GSK, Boehringer Ingelheim, MSD and Schering Plough, funds for consultancy from GSK, Pharmacia and Chiesi Pharmaceuticals and travel grants from GSK, Boehringer Ingelheim, AstraZeneca, Chiesi Pharmaceuticals and MSD.

\section{References}

1. Health Department of Great Britain. General Practice in the NHS: A new Contract 1989.

2. Jewell $D$, Turton $P$. What's happening to practice nursing? Moves towards greater professionalism should be supported. BMJ 1994;308:735-6.

3. Atkin K, Lunt N, Parker G, Hirst M. Nurses Count: a national census of practice nurses. 1-147. 1993. University of York.

4. Atkin K, Lunt N. Negotiating the role of the practice nurse in general practice. J Adv Nurs 1996;24(3):498-505.

5. Martin J. The Next Generation. http://www.wipp.nhs.uk/uploads/ jeanett_martin_article.doc.2007. Accessed 03/07/2007.

6. NHS Management Executive. GP contract health promotion package: guidance on implementation. 3. 1993. Department of Health, FHSL.

7. The COPD Guidelines Group of the Standards of Care Committee of the BTS. BTS guidelines for the management of chronic obstructive pulmonary disease. Thorax 1997; 52 Suppl 5:S1-28.

8. National Health Service Confederation and British Medical Association. The New General Medical Services Contract. 2003. London, Department of Health.

9 Department of Health. Chronic Obstructive Pulmonary Disease National Service Framework. http://www.dh.gov.uk/en/Policyandguidance/Healthandsocial caretopics/DH_4138532.2007. Accessed 03/08/2007.

10. Barnes G, Partridge MR. Community asthma clinics: 1993 survey of primary care by the National Asthma Task Force. Qual Health Care 1994;3:133-6.

11. Department of Health. Statistics for general medical practitioners in England: 1994-2004. 2005. London: $\mathrm{DoH}$.

12. Majeed A. The future of single-handed general practices. BMJ 2005; 330(7506):1460-61.

13. Eastwood AJ, Sheldon TA. Organisation of asthma care: what difference does 
J Upton et al.

it make? A systematic review of the literature. Qual Health Care 1996; 5(3):134-43.

14. Pilotto LS, Smith BJ, Heard AR, McElroy HJ, Weekley J, Bennett P. Trial of nurserun asthma clinics based in general practice versus usual medical care. Respirology 2004;9(3):356-62.

15. The NHS Improvement Plan. www.dh.gov.uk/publications 2004. Accessed 03/07/2007

16. Dickinson J, Hutton S, Atkin A, Jones K. Reducing asthma morbidity in the community: the effect of a targeted nurse-run asthma clinic in an English general practice. Respir Med 1997;91(10):634-40.

17. RCGP. General Practice in the UK: A basic overview. http://www.rcgp.org.uk/
pdf/ISS_INFO_04_MAY05.pdf.2005. Accessed 16/07/2007.

18. Hippisley-Cox J, Pringle M, Coupland C, Hammersley V, Wilson A. Do single handed practices offer poorer care? Cross sectional survey of processes and outcomes. BMJ 2001;323(7308):320-23.

19. National Institute of Clinical Excellence. Chronic obstructive pulmonary disease: national clinical guideline for management of chronic obstructive pulmonary disease in adults in primary and secondary care. Thorax 2004;59(Suppl 1).

20. Guest JF. The annual cost of Chronic Obstructive Pulmonary Disease to the UK's National Health Service. Disease Management \& Health Outcomes 2007;5(2): 93-100.

\section{Available online at http://ww w.thepcrj.org}

